\title{
MedienPädagogik
}

Zeitschrift für Theorie und Praxis der Medienbildung

Jahrbuch Medienpädagogik 3.

Zweitveröffentlichung aus: Jahrbuch Medienpädagogik 3. (2003) Opladen: Leske + Budrich. Herausgegeben von Ben Bachmair, Peter Diepold und Claudia de Witt.

\section{Medien - Generationen - Wissen \\ Überlegungen zur medienpädagogischen Forschung - dargestellt am Beispiel der Frage nach dem Weltwissen globaler Mediengenerationen}

\section{Theo Hug}

Mit den aktuellen gesellschaftlichen, technologischen und politischen Entwicklungen haben sich neue Fragen und Aufgabenbereiche für die Medienpädagogik ergeben. Diese werden im vorliegenden Beitrag skizziert, wobei in Abgrenzung von traditionellen Auffassungen für ein weiteres Verständnis von Medienpädagogik argumentiert wird. Die Erweiterung des thematischen Horizonts wird anhand einer Pilotstudie zur Frage des Weltwissens von Mediengenerationen exemplarisch verdeutlicht. Abschließend werden einige Überlegungen zur Medienkompetenz, der Problematik diesbezüglicher Verkürzungen und deren Stellenwert im Lichte des „medial turn“ zur Diskussion gestellt.

\section{Aktuelle Entwicklungen und neue Fragen an die Medienpädagogik}

Im Zuge der Beschreibung aktueller sozialer Veränderungsprozesse hat sich das Spektrum der Bindestrich-Gesellschaften erheblich erweitert. Seit einigen Jahren finden dabei immer öfter die Stichworte „Informationsgesellschaft“, „Wissensgesellschaft“, „Kommunikationsgesellschaft“ oder „Mediengesellschaft“ Verwendung. Sie tauchen einerseits in verschiedenen Wissenschaftszweigen, andererseits aber auch in Zeitungen, Rundfunk und Fernsehen auf, und sie werden im Alltagsleben mitunter so selbstverständlich gebraucht wie Telefon, Fax oder neuerdings E-Mail. Unter diesen Stichworten sind also differenzierte Beschreibungen sowie alltagspraktische Vereinfachungen anzutreffen, kurzum: sie figurieren in verschiedenen Diskurszusammenhängen und sie nehmen in der einen oder anderen Weise Bezug auf die neueren computertechnologischen Entwicklungen. Entsprechend haben sich auch die Aufgabenbereiche der Medienpädagogik erheblich erweitert, beispielsweise im Hinblick auf Fragen nach 
- der Veränderung von Kommunikationskulturen und Optionen der Entwicklung sozialer Verantwortung im Zusammenhang steigender Mediennutzungszeiten,

- den Möglichkeiten der gelingenden Verknüpfung von emotionalem und sozialem Lernen mit informationstechnischer Bildung,

- der individuellen und gesamtgesellschaftlichen Bedeutung von medialen Gewaltdarstellungen im Spannungsfeld von Wirklichkeit, Imagination und Fiktion,

- der Relevanz der Werbeindustrie für die Alltagskultur und das Konsumverhalten von Kindern, Jugendlichen und Erwachsenen,

- den Konsequenzen der massenhaften Verbreitung trivialer Medienangebote und den Optionen differenzierter Mediennutzung,

- der Bedeutung der Ergebnisse der Wissenskluft-Forschung und den diesbezüglichen Konsequenzen für die Bildungsplanung und -politik,

- tragfähigen Konzepten der Medienkompetenz im Hinblick auf ein gedeihliches Zusammenspiel kommunikativer, medialer und interkultureller Kompetenzen und entsprechenden Aussichten auf die tendenzielle Beförderung sozialverträglicher Entwicklungen,

- der Veränderung der Lern- und Kommunikationsverhältnisse im Lichte aktueller Individualisierungs- und Globalisierungsprozesse.

Diese und viele andere Fragen (vgl. Hug 2002, S. 193 f.) fallen allesamt primär oder zumindest anteilig in die Zuständigkeit der Medienpädagogik. Dieter Baacke spricht in diesem Zusammenhang von einem Expansionskurs, der eine Beantwortung dieser Fragen auf der Basis grundlegender Perspektiven nahe legt (vgl. Baacke 1997, S. 3). Das bedeutet unter anderem, dass es nicht nur um absichtsvolle, mehr oder weniger wohldesignte Lernarrangements gehen kann, die in den Augen so mancher Bildungsverantwortlichen das „eigentliche“ Lernen ausmachen. Zunehmend wichtiger wird die Auseinandersetzung mit den „informellen“ und zum Teil unbewussten Lernprozessen, die unsere Denk- und Wahmehmungsgewohnheiten grundlegend strukturieren.

Die aufgeworfenen Fragen machen darüber hinaus die Ausweitung ihres Gegenstandsbereichs über ältere Auffassungen der Konzentration auf schulische, unterrichtstechnologische oder etwa jugendschützerische Dimensionen deutlich. Sie zeigen, dass die Erweiterung der Fragehorizonte und Problemstellungen unter fachimmanenten Gesichtspunkten nicht angemessen erklärt werden kann. Diese Erweiterungen ergeben sich vielmehr im Zuge der gesellschaftlichen und technologischen Veränderungsprozesse und die Fragen werden häufig von „außen“ an die Medienpädagogik herangetragen.

\section{Plädoyer für eine zeitgemäße Medienpädagogik}

Angesichts der Vielfalt und des Aspektreichtums dieser Fragen und der damit verbundenen Aufgabenbereiche liegt die Vermutung der Überforderung der 
Medienpädagogik nahe. Wer intensiver in die gegenwärtigen Auseinandersetzungen um die Möglichkeiten und Grenzen der Medienpädagogik involviert ist, wird in diesem Zusammenhang gelegentlich mit „Alles-oder-Nichts“-Argumentationen konfrontiert. Das bedeutet, dass auf der einen Seite große Hoffnungen in die Medienpädagogik gesetzt werden: Nachdem immer mehr Menschen in Informations- und Kommunikationsgesellschaften hineinwüchsen, der gesteigerte Medienkonsum alle Altersgruppen betreffe und die zunehmende Vermengung privater, öffentlicher und beruflicher Mediennutzungsformen offenkundig geworden sei, müsse die Medienpädagogik gleichsam zu dem zentralen Umschlagplatz aller medienwissenschaftlichen und medienpraktischen Bemühungen werden. In diesen Argumentationen spielt die Metapher der „Durchdringung“ aller Lebensbereiche, Wahrnehmungsformen, Sozialisations- und Lernprozesse durch die Medien häufig eine tragende Rolle. Andererseits wird die Zuständigkeit der Medienpädagogik für die skizzierten Fragen und Problembereiche auch massiv infragegestellt, und zwar unter Hinweis auf den reaktiven Charakter ihrer Bemühungen auf bereits vorhandene Entwicklungen, die in erster Linie dem Diktat ökonomischer und nicht dem emanzipatorischer Interessen folgen, auf die Unsinnigkeit ihres Betreuungsanspruchs im globalen Spiel der Medienkonzerne, auf die generelle Bedeutungslosigkeit ihrer veralteten Vorstellungen der Überwachung und der Bewahrung vor schädlichen Einflüssen, manchmal auch unter Verweis auf den ohnedies stattfindenden, informellen Kompetenzerwerb von Kindern und Jugendlichen. Nachdem sich die Aufwendungen für Bildungskonzepte und -materialen bestenfalls in der Größenordnung von Zinsen der sogenannten „Technologiemilliarden“ bewegen, seien ohnedies keine gesellschaftlichen Effekte der medienpädagogischen Bemühungen zu erwarten. Damit sei die Medienpädagogik obsolet und ihr emanzipatorischer Traum ausgeträumt.

Wie für die Erziehungswissenschaft und Pädagogik insgesamt gilt für die Medienpädagogik erst recht: sie wird einerseits gerne maßlos überschätzt und andererseits nicht gerade selten unterschätzt, gelegentlich auch für bedeutungslos erklärt. Beide Extreme verkennen ihre Möglichkeitsräume: Das eine, weil es der Medienpädagogik generell die Prophylaxe und Therapie unerwünschter Effekte der Mediensozialisation und kommerzialisierten Medienkommunikation zumutet, das andere, weil es die Potentiale der kritisch-reflexiven Gestaltung und Erweiterung von Handlungsspielräumen unterbewertet. Ihre Möglichkeiten und Zuständigkeiten liegen aber gerade in der Analyse, Erkundung und Gestaltung dieser Spielräume. Dass sie dabei in manchen Hinsichten nur sehr vorläufige Vermutungen, in anderen nur skizzenhafte Antworten, in den seltenen Fällen fundierte Untersuchungsergebnisse und so gut wie keine „Rezepte“ vorweisen kann, liegt auf der Hand, wenn man die Breite der Aufgabenbereiche ${ }^{1}$ bedenkt. Da hilft auch keine Redukti-

1 Diese Aufgabenbereiche werden im Allgemeinen in die vier Teilbereiche Medienerziehung, Mediendidaktik, Medienkunde, Medienforschung gegliedert (vgl. Issing 1987, 24ff; v. Wensiersky 1995, 160; Baacke 1997, 4; Merkert 1997, 1058ff; Sinhart-Pallin 1997, 389ff; Hug 2000). Es wird sich zeigen, ob und inwieweit diese Einteilung in Zukunft noch sinnvoll sein wird, da im Zuge der wissenschaftlichen, gesellschaftlichen und 
on der Zuständigkeiten im Sinne eines engen Verständnisses von Medienpädagogik.

\begin{tabular}{ll}
\hline Medienpädagogik im engeren Sinne & Medienpädagogik im weiteren Sinne \\
\hline $\begin{array}{l}\text { Primär praxisorientierte Zugänge in pädagogi- } \\
\text { scher Absicht }\end{array}$ & $\begin{array}{l}\text { Handlungsorientierte und theoretisch motivier- } \\
\text { te Zugänge }\end{array}$ \\
$\begin{array}{l}\text { Konzentration auf den pädagogischen Bezug } \\
\text { (im engeren Sinne) sowie auf interaktioneile } \\
\text { oder unterrichtstechnologische Dimensionen }\end{array}$ & $\begin{array}{l}\text { Berücksichtigung kontextueller, lebensweltli- } \\
\text { cher, massenkommunikativer, kultureller, öko- } \\
\text { nomischer, politischer und gesellschaftlicher } \\
\text { Dimensionen }\end{array}$ \\
Konzentration auf Kinder und Jugendliche & $\begin{array}{l}\text { Berücksichtigung aller Altersgruppen im Sinne } \\
\text { einer Pädagogik als „Lebenslaufwissenschaft“ }\end{array}$ \\
$\begin{array}{l}\text { Beschränkung auf pädagogische Binnentradi- } \\
\text { tionen }\end{array}$ & $\begin{array}{l}\text { Favorisierung Interdisziplinärer Ansätze und } \\
\text { transdisziplinärer Zugänge }\end{array}$ \\
$\begin{array}{l}\text { Rückgriff auf wesensanthropologische Konzep- } \\
\text { tionen (Menschenbildpädagogik) }\end{array}$ & $\begin{array}{l}\text { Anthropologische Offenheit bzw. Orientierung } \\
\text { an Konzepten der historisch-kritischen Anthro- } \\
\text { pologie }\end{array}$ \\
Geschlechtsblinde Konzeptionen & $\begin{array}{l}\text { Berücksichtigung geschlechterdifferenzieren- } \\
\text { der Fragestellungen }\end{array}$ \\
Beschränkung auf regionale, nationale oder & $\begin{array}{l}\text { Berchtigung regionaler und (sub-)kultu- } \\
\text { reller Perspektiven wie auch transnationaler, } \\
\text { globaler und interkultureller Perspektiven }\end{array}$ \\
\hline
\end{tabular}

Tab. 1.: Charakteristika enger und weiter Verständnisse von Medienpädagogik.

Eine Medienpädagogik, die sich auf personale und curriculare Dimensionen konzentriert (vgl. Merkert 1992, 2 und 56ff), bekommt meines Erachtens die zeitgenössischen Probleme nicht in adäquater Weise in den Blick. Für zielführender halte ich eine Auffassung von Medienpädagogik, die auf ideologische Fixierungen und disziplinäre Verengungen verzichtet und statt dessen im Rückgriff auf sozial- und kulturwissenschaftliche Denkangebote die Schnittstellen des Aufwachsens, der Arbeit, der Bildung, der Erziehung, des Lernens und der Verständigungsprozesse zu den medialen Entwicklungen bearbeitet (vgl. Baacke 1997, S. 5). Die Konkretisierung von Fragestellungen, Problemformulierungen und Blickrichtungen erfolgt dabei weniger im Lichte pädagogischer Traditionen oder einer immanenten Logik der Pädagogik, sondern vielmehr auf dem Hintergrund des gesteigerten gesellschaftlichen Bedarfs an medienbezogenen Reflexions- und Orientierungsangeboten

technologischen Entwicklungen ständig neue Aufgaben, Konzepte und Verfahren hervorgebracht werden (vgl. Rickert 1993). Die Medienpädagogik ist damit in einem Spannungsfeld angesiedelt, in dem die Zuständigkeiten fließend sind und im Detail immer wieder neu abgesteckt und formuliert werden müssen (vgl. die Stichworte ,ästhetische Bildung', ,informationstechnische Bildung', ,sozialwissenschaftliche Informatik', ,Medienökologie', ,Instruktionsdesign' sowie ,Informations-', ,Wissens-` und ,Kulturmanagement'). 
sowie in Auseinandersetzung mit anderen medienwissenschaftlichen Bemühungen. Eine Schwierigkeit besteht dabei darin, dass sie sich nicht mit wissenschaftlichen oder philosophischen Betrachtungen allein begnügen kann, sondern auch didaktische Konzeptionen, Reflexionshilfen und Orientierungsangebote für praktischpädagogische Zwecke bereitstellen muss. Darüber hinaus dürfte aber die größte Herausforderung im angemessenen Umgang mit komplexen Problemlagen liegen. Jede Medienpädagogik, die sich nicht von vornherein dem techno-ökonomischen Diktat der Medienfröhlichkeit unterordnen oder der Entwicklung von Strategien der Gewissensberuhigung für Technokraten verschreiben will, wird um eine differenzierte Auseinandersetzung mit medienkritischen und sozialwissenschaftlichen Denkangeboten nicht herumkommen. Darin liegen zugleich ihre Chancen der Professionalisierung und der kritisch-reflexiven Technologiegestaltung (vgl. Hug 1998).

\section{Konsequenzen für die Forschung - dargestellt am Beispiel der Frage nach dem Weltwissen globaler Mediengenerationen}

Wie kann die Medienpädagogik den erweiterten Anforderungen und Spielräumen Rechnung tragen? Welche Konsequenzen ergeben sich daraus für die Forschung? Eine detaillierte Antwort auf diese Fragen würde Buchbände und Web-Seiten füllen. Ich möchte am Beispiel der Frage nach dem Weltwissen globaler Mediengenerationen aufzeigen, wie insbesondere das Zusammenspiel lokaler und globaler Dimensionen Berücksichtigung finden kann.

Dass das Aufwachsen in unterschiedlichen Medienlandschaften von zentraler Bedeutung für die Ausbildung von Orientierungsmustern, Deutungsschemata und Mediennutzungsformen darstellt, ist bereits eine Alltagserfahrung. Dies zeigt sich beispielsweise am spielerischen Umgang der heute Heranwachsenden mit den sogenannten „Neuen Medien“. Mancherorts ist von einer Netz-Generation die Rede, die im Unterschied zur Fernseh-Generation selbstbewusster und flexibler ist. Denken wir beispielsweise an den schulischen Unterricht und die Schwierigkeiten, die viele „printsozialisierte“ Lehrerinnen haben, wenn sie Internet-Angebote für Bildungszwecke fruchtbar machen wollen. Die Chancen für gelingende Lernprozesse sind hier gering, wenn nicht Bedacht auf die Rezeptionsformen der neuen Medien-Generation im „New Age of Visual Thinking“ (vgl. Baacke 1999) genommen wird. Meines Erachtens lassen sich solche Schwierigkeiten nur dann angemessen nachvollziehen, wenn wir in Rechnung stellen, dass sich die entscheidenden Eindrücke einer jeweiligen Generationsbiographie über frühe Leseerfahrungen, audiovisuelle Medien und Musik und nicht zuletzt über die Wahrnehmung medienvermittelter, politischer Ereignisse bestimmen. Jede dieser Generationen bildet unterschiedliche Zugänge zur Welt, Relevanzkriterien, Orientierungsmuster und Gefühle des „In-der-Welt-Seins“ - kurzum: unterschiedliche Formen des „Weltwissens“ aus. 
Was ist damit gemeint? Was heißt überhaupt „Weltwissen“? Der Ausdruck „Weltwissen“ scheint meinen Recherchen zufolge in der wissenschaftlichen Literatur erst seit den 80er Jahren auf. Freilich, Fragen nach der Erkennbarkeit von Welt sind so alt, wie die menschliche Möglichkeit Fragen zu stellen. Die entsprechenden Antworten, soweit sie sich auf grundlegende Anschauungen oder deren unaussprechliche Hintergründe beziehen, können in einem weiten Sinne als Versionen von „Weltwissen“ aufgefasst werden. „Weltwissen“ kann darüber hinaus konkreter konzeptualisiert werden als

- Orientierungswissen im Lichte von Weltanschauungen,

- „Welt des Wissens“ im Sinne von Poppers (1973) Welt 3 der objektiven Gedankeninhalte,

- Hintergrundwissen oder als implizites Wissen (vgl. Polanyi 1985),

- Alltagswissen oder als lebensweltlicher Wissensvorrat (vgl. Schütz/Luckmann 1979, 1984),

- Set konzeptueller Annahmen und Vorstellungen über Regularitäten in unserer Welt (vgl. Rusch 1987, S. 243),

- kollektives Wissen (vgl. Schmidt 1994, S. 236ff),

- gesellschaftlich geteilte Haltungen, die das semantische Gedächtnis einer Gesellschaft ausmachen, das von den Einzelnen mittels kognitiv verankertem Kontrollsystem in konkreten Situationen in Anschlag gebracht wird (vgl. van Dijk 1987),

- Zeitschemata, kognitive Landkarten und Akteurvorstellungen über die Eigenschaften und das Verhalten bestimmter Personengruppen (vgl. Winterhoff-Spurk 1989),

- kognitionswissenschaftliche Modelle globaler konzeptueller Kategorien und Schematheorien (vgl. Rickheit 1993).

Diese Begriffe rücken jeweils unterschiedliche, zum Teil auch ähnliche Gesichtspunkte in den Vordergrund. Wenn wir davon ausgehen, dass die Mediensozialisationserfahrungen eine entscheidende Rolle bei der Ausbildung des Weltwissens spielen, dann stellt sich die Frage, wie sich solche Prozesse in unterschiedlichen Generationen und Kulturen abspielen und welche Unterschiede und Gemeinsamkeiten sich beschreiben lassen. Diese Thematik wird im Forschungsprojekt Globale Mediengenerationen 2000 bearbeitet. Sehen wir zu, was „Weltwissen“ im Kontext dieses Projekts meint.

Die aktuellen Medienentwicklungen und mehr noch der intensivierte Wettbewerb um Aufmerksamkeit und Anerkennung bringen Veränderungen in der individuellen und gesellschaftlichen Wissensorganisation mit sich. Mit den neuen Formen des „In-derWelt-Seins“ korrespondieren neue Formen des Weltwissens. So wird an der Schwelle zum 21. Jahrhundert deutlich, dass Faktoren wie das Zusammenwachsen der Märkte, die Satellitenkommunikation, die zunehmende Mobilität und die überregionalen Dimensionen verschiedener Risiken und Krisen nach und nach ein neues Bewusstsein globaler Vernetzung und „virtueller Gemeinschaft“ ermöglicht haben. Dieser Zusammenhang wird im Forschungsprojekt Globale Mediengenerationen 2000 im 
Zuge einer Analyse von Medienereignissen des 20. Jahrhunderts als Bausteine kollektiver Erinnerungen untersucht. Ziel ist u.a. die Beschreibung der Wissensarchitektur verschiedener globaler Generationen im familialen und interkulturellen Kontext. In Abgrenzung von älteren Deutungen einer globalen Vereinheitlichung, die von der „Welt“ im Sinne eines homogenen Ganzen ausgingen, werden dabei jene Dimensionen herausgearbeitet, die der Gleichzeitigkeit und Verhältnismäßigkeit parallel verlaufender Globalisierungsprozesse Rechnung tragen (vgl. Robertson 1992). Entsprechend geht es bei der Analyse der Rezeption und Verarbeitung globaler Medienereignisse darum, die „Gleichzeitigkeit der Ungleichzeitigkeit“ im Zusammenspiel lokaler, kulturspezifischer und globaler Dimensionen zu reflektieren.

Diese Ausgangslage wurde bereits exemplarisch im Zusammenhang einer qualitativen Pilotstudie illustriert und weiter konkretisiert (vgl. Volkmer 1998). Diese Studie hatte zum Ziel, explorativ einen Ansatz zu finden, um Generationen in bezug auf Dimensionen der Kollektivität innerhalb spezifischer biographischer Stadien zu beschreiben, in denen Weltwissen als wichtiges Element von Generationenidentität aufgebaut wird. In dieser Pilotstudie, die 1993 an der Universität Bielefeld durchgeführt wurde, wurden drei Studenten und vier Studentinnen aus Marokko, Japan, Türkei, China, Korea und Taiwan befragt. Die Fragestellungen richteten sich darauf, inwiefern - trotz erheblicher räumlicher und kultureller Distanz - dieselben medial vermittelten Ereignisse in einem gemeinsamen „Lebenszeitraum“ Kindheit erinnert werden. Neben dieser zentralen Frage sollten auch die Unterschiede, die speziellen Wissensprofile, innerhalb der unterschiedlichen, durch die Interviewten repräsentierten Weltregionen deutlich werden. Im Zuge dieser Befragung stellte sich u.a. heraus, dass ein kollektives Weltwissen durch zwei Nachrichtenereignisse getragen wird: Kennedy's Ermordung und die erste Mondlandung.

„Neben diesen ,globalen` Medienereignissen, die von fast allen Befragten erinnert werden, sind die nachgefragten Welt-Ereignisse des Internationalen Almanach nur an der Peripherie dieser ,Nachrichtengeneration`a angesiedelt. Den Befragten sind zwar häufig die Namen der an den nachgefragten Nachrichtenereignissen Beteiligten bekannt, sie haben dieses Wissen jedoch zeitversetzt, in einer biographisch späteren Lebensphase (z.B. während des Studiums) erworben. Genaueres Nachfragen nach dem Kontext Wissen zeigte, dass selbst Fragmente des Ereignisses nur vage bekannt sind und der weitere Ereignisverlauf zum Teil nur grob referiert werden kann“ (Volkmer 1998, S. 176).

Für das laufende internationale Kooperationsprojekt Globale Mediengenerationen $2000^{2}$ wurde im Zuge der Vorarbeiten folgende Ereignistafel zusammengestellt, die jeweils noch regionenspezifische Ergänzungen erfährt:

2 Das Projekt wird gegenwärtig unter der Leitung von Ingrid Volkmer und unter Mitar beit von 14 MedienwissenschaftlerInnen aus aller Welt durchgeführt. Eine Publikation mit den Ergebnissen wird voraussichtlich beim Verlag Peter Lang 2003 erscheinen. 


\begin{tabular}{lll}
\hline Events during 1935-1945 & Events during 1965-1975 & Events during 1989-1999 \\
\hline War in Spain & Photos of Mars & Gulf War \\
Abdication of Edward Vlii & Cultural Recognition in China & Fall of Eastem Block \\
Berlin Olympics & 1968 Student Revolutions & Beijing Massacre \\
Kristallnacht & Prague Spring & Reunification of Germany \\
Beginning of World War II & Independence movements in & Economic Crisis in Asia \\
& Africa & \\
Salt March in India & OPEC Crisis & Princess Diana's death \\
Fall of Singapore & Vietnam & Liberation of Nelson Mandela \\
& & /South Africa's retum to inter- \\
& & national fold \\
Pearl Harbour & Rise of PLO & OJ's trial /Rodney King \\
Atom Bombs & Watergate & Closer - European Monetary \\
Auschwitz & & - Union \\
\hline
\end{tabular}

Tab. 2.: Ereignistafel im Projekt Globale Mediengenerationen 2000.

Hinsichtlich der Altersstruktur wurden auf dem Hintergrund medientechnischer Entwicklungen sowie auf der Basis von Vorvermutungen über die Relevanz der aufgelisteten Ereignisse drei Gruppen ausgewählt:

\begin{tabular}{lll}
\hline Gruppe & Alter (im Jahr 1999) & mit Bezug auf die Jahre \\
\hline 1 & $70-35$ & $1935-1945$ \\
2 & $40-45$ & $1965-1975$ \\
3 & $15-20$ & $1989-1999$ \\
\hline
\end{tabular}

Tab. 3.: Altersstruktur der untersuchten Gruppen im Projekt Globale Mediengenerationen 2000.

In der Untersuchung, die parallel in elf Ländern auf allen Kontinenten durchgeführt wird, geht es nun darum, herauszufinden, inwieweit die Medienereignisse Bestandteil biographischer Erinnerungen der Befragten beschrieben werden können. Dabei gehen wir davon aus, dass sich Generationen nicht nur formal als bevölkerungsstatistischer Durchschnittsabstand zwischen den Geburtsjahren der Eltern und ihrer Kinder (25 bis 30 Jahre) oder inhaltlich als Aggregat benachbarter Altersgruppen mit charakteristischen Verhaltensmerkmalen beschreiben lassen, die sich jeweils von anderen Altersgruppen unterscheiden. Als verbindendes Element von Generationen fungieren auch die jeweiligen Vorstellungen von Raum und Zeit innerhalb einer Weltkommunikationsgesellschaft, die zunehmend von Bildern weltweit verbreiteter Ereignisse bestimmt werden.

„Die Vorstellung von ,Zeit` und ,Raum` als verbindendes Element von Generationen ist innerhalb einer Weltkommunikationsgesellschaft, um eine These im Kontext der anfänglichen Debatte zu formulieren, zu einem bestimmten, zunehmenden Grad von ,Images` weltweit verbreiteter Ereignisse 
bestimmt. Medienereignisse sind innerhalb biographischer Phasen einer Weltgeneration eingelagert und prägen das Wissen um globale Vergangenheit und Gegenwart in einem somit generationsspezifisch geprägten Blick auf die ,Welt-Realität"“ (Volkmer 1998, S. 170). In der erwähnten explorativen Bielefelder Studie hat sich gezeigt, dass Medienereignisse offensichtlich in Interpretationskategorien erinnert werden, „die zum Teil ideologisch, zum Teil kulturell spezifisch oder sozial sind. Dieses bedeutet, dass Medienereignisse [...] mit einem bestehenden Wissenskontext verkoppelt erinnert werden. Diese Beobachtung gilt im Rahmen der durchgeführten Studie für Weltereignisse aber auch für Ereignisse mit nationalem Charakter. Die Befragten (außer die Japanerin, die bereits mit einer kindlichen Medienwelt aufgewachsen ist) berichten über ein reges politisches Familienklima (vermittelt über das politische Interesse der Eltern bzw. des Vaters), das sie als Kinder bereits wahrgenommen haben. In dieser ersten ,Fernsehgeneration` ist die Zeitung (ergänzt durch Radio und in wenigen Fällen Fernsehen) das Leitmedium der Kindheit“ (Volkmer 1998, S. 176).

Für die erinnerte Wirklichkeit ist also einerseits der soziale Kontext der Rezeption relevant. Andererseits ist insbesondere der mediale Kontext bedeutsam, und zwar sowohl im Hinblick auf die jeweils verfügbaren Kommunikationsmedien als auch im Hinblick auf den jeweiligen Zugang. Entsprechend spielen als „Leitmedien“ Zeitungen, Zeitschriften, Printmedien, Radio, Fernsehen, Multi-channel-colour-TV sowie das Internet eine unterschiedliche Rolle. Volkmer beschreibt die verbindenden Elemente einer Generation im sozialen Umfeld der Rezeptionssituation in Anlehnung an Stephen und Howe als „Life Marker“, die sich im Umfeld einer Generation synchron oder asynchron abbilden lassen: „Dieser Begriff erlaubt es, die kulturellen, sozialen und politischen Spezifitäten im Umfeld eines Nachrichtenereignisses im subjektiven biographischen Blick einzubeziehen. Für jeden der Befragten existieren subjektive medial geprägte ,Life Marker"“ (Volkmer 1998, S. 178).

Bei der Auswertung der Gruppendiskussionsprotokolle im Projekt Globale Mediengenerationen 2000 wird sich zeigen, inwieweit die Antworten im Sinne vereinheitlichender und differenzierender Wissensbestände von „Welt“-Generationen zu verorten sein werden und inwieweit dieses Wissen im Sinne von „Weltwissen“ als wichtiges Element von Generationenidentität konzipiert werden kann. Des weiteren stellt sich die Frage, inwieweit sich das hier anvisierte Weltwissen angesichts seiner fragmentarischen, Simplexen, spontan orientierenden und leicht verständlichen Züge als Variante des Instantwissens (vgl. Hug 1998) begreifen lässt. Das scheint naheliegend zu sein, da die „Weltwissensfragmente“ den Charakter von Schlüsselwörtern und Stereotypen aufweisen, die einen globalen Zeitrahmen der Parallelität von Handlungen suggerieren und die auf diese Weise einen Rahmen für das Image einer globalen Gemeinschaft herstellen.

Andererseits dürfte das allerdings noch nicht der Weisheit letzter Schluss sein, denn dieses Weltwissen lässt sich nicht nur im Sinne eines flüchtigen „Sofort-Wissens“ beschreiben. Es hat vermutlich ein höheres Reflexionspo- 
tential als das „unproblematische Gebrauchswissen“. Als verdichtetes, „sedimentiertes“ Wissen kann es auch als handlungsleitendes „Hintergrundwissen“ beschrieben werden. Das Weltwissen globaler Mediengenerationen wäre dann jener Teil der kollektiven, konzeptuellen Annahmen und Vorstellungen (Rusch 1987, S. 243), der für bestimmte Generationen eine ausgezeichnete Rolle spielt. Wenn das „allgemeine Weltwissen“ als Inbegriff unserer Vorstellungen von der „wirklichen“ Beschaffenheit der Welt unsere Konzepte von Objekten, Zuständen, Ereignissen und deren Folgen sowie unsere Vorstellungen von Raum und Zeit konzipiert, dann besteht das Weltwissen der Generationen in jenen medienvermittelten, partiell „gleichgeschalteten“ Wirklichkeitsvorstellungen und kulturspezifischen Vereinheitlichungen, die das Grundgefühl des „In-der-Welt-Seins“ einer Generation, deren Verständnis von Regularitäten in der Welt sowie deren Modalitäten der Unterscheidung von relevanten und nicht-relevanten Ereignissen ausmachen. Weltwissen lässt sich damit im Sinne einer Figur-Grund-Relation in doppelter Weise konzipieren, oder - in einem anderen, sportiven Bild ausgedrückt: Weltwissen kann sowohl als „Standbein“ als auch als „Spielbein“ betrachtet werden. Das „Spielbein“ wäre dann das fragmentarische Sofort-Wissen von globalen Medienereignissen, und das „Standbein“ wäre das konzeptuelle Hintergrundwissen, das die thematischen und medialen Horizonte einer Generation ausmacht und das die typischen Wiesen der Herstellung von Wirklichkeitsbezügen, Bedeutsamkeiten und Orientierungsmustem beinhaltet. Dieses Hintergrund- oder Common-Sense-Wissen ist also nicht im Sinne eines für beide Geschlechter allerorts und für alle Zeiten gültigen „allgemeinen Weltwissens“ zu verstehen. Es ist vielmehr ein gemeingültiges Wissen, das in der relationalen Verknüpfung unterschiedlicher Perspektiven gründet und zwar ohne Rückgriff auf ein noch einmal dahinterliegendes, „festes Fundament“ und ohne Auflösung dieser Perspektiven in einen frei „flottierenden Zeichensalat“.

\section{Ausblick}

Wer die Welten unterschiedlicher Medien-Generationen und die damit verbundenen Arten und Weisen der Ver- und Entschlüsselung nachvollziehen will, wird nicht umhin kommen sich mit der Frage der Medienkompetenz zu befassen. Sie scheint hier wie auch in der neueren medienpädagogischen Diskussion insgesamt die Schlüsselfrage darzustellen. „Medienkompetenz“ erweist sich gleichsam als Drehtürbegriff, der sowohl in wissenschaftlichen als auch in wirtschaftlichen, politischen und pädagogischen Kontexten Verwendung findet. Dabei wurde seit dem ersten Anstoß von Baacke in den Siebzigern (vgl. Baacke 1973; Lauffer/Volkmer 1995) eine Reihe von Konzepten entwickelt, die Kompetenzbereiche akzentuieren, wie insbesondere die Befähigung 
— zum „mündigen Rezipienten“ im Sinne individueller und demokratischer Orientierung,

- zur Wahrnehmung technischer Bilder und zum reflexiven Umgang mit neuen Codes und medialen Symbolen,

- zum aktiven Mediennutzer, der Entwicklungs- und Gestaltungsmöglichkeiten auslotet,

- zur kritischen Reflexion ethischer, ökonomischer, interkultureller, politischer, geschlechtsspezifischer, sozialer oder juristischer Aspekte der Informations- und Kommunikationstechnologien,

- zur Informationsbewältigung, Wissensorganisation und zum Medienmanagement,

- zum emanzipatorisch motivierten Medienanbieter oder

- zur verantwortungsbewussten Integration pädagogischer, sozialpolitischer und ökonomischer Motive im Lichte erziehungs- und sozialwissenschaftlicher Befunde und technologischer Optionen.

Diese Kompetenzbereiche werden unterschiedlich gebündelt und gewichtet (vgl. exemplarisch Baacke 1997, S. 98ff; Bayerische Landeszentrale 1996; Enquete-Kommission 1997; Lauffer/Volkmer 1995; Moser 1995, S. 215f.; v. Rein 1996; Schwarzer 1998, 15f.; Schorb 1998, S. 20ff; Schorb 1999). Was das Grundverständnis der diversen Bündelungen und Gewichtungen betrifft, sind allerdings immer wieder Verkürzungen anzutreffen. So hat insbesondere Baacke betont, dass Medienkompetenz im Sinne einer gesellschaftlichen Partizipationskompetenz verstanden und weder subjektivistisch noch rationalistisch verkürzt werden soll (vgl. Baacke 1997, S. 99). Abgesehen von der häufigen Vernachlässigung der Körperlichkeit und der Emotionalität der Menschen sind auch Bedenken im Hinblick auf die folgenden Verkürzungen angebracht:

Auffallend ist, dass die Vorstellungen von Medienkompetenz häufig auf einzelne - seien es technologische, ökonomische, kritische, sozial-kommunikative oder pädagogische - Dimensionen verkürzt werden, und dass diese Verkürzungen nicht selten Hand in Hand mit tendenziellen Verabsolutierungen der jeweiligen Perspektiven geht. Unter Beschränkung auf praktische Medienarbeit, Einübung in Programmlogiken, kritische Reflexion, ökonomische Orientierung, sozialwissenschaftliche Theorie oder ästhetische Darstellung lässt sich aber keine Medienkompetenz entfalten, die den komplexen individuellen und gesellschaftlichen Problemlagen gerecht werden könnte. Die Artikulation solcher Dimensionen erfolgt immer wieder auf dem Hintergrund eines unreflektierten Komponentenmodells. Die additive Auflistung von Fähigkeiten und Zuständigkeiten vernachlässigt dynamische Perspektiven und suggeriert irreführenderweise die Austauschbarkeit der Komponenten. Für das Gelingen der symbolischen Austauschprozesse erscheint allerdings die integrative Entwicklung sozial-kommunikativer, technischer, theoretischer, methodologischer, selbstreflexiver Kompetenzdimensionen in Relation zu Lebensabschnitten, sozio-kulturellen Problemlagen und spezifischen Anwen- 
dungskontexten erforderlich. Dabei lässt sich eine allgemeine Medienkompetenz von einer speziellen unterscheiden: Während erstere auf die mediale Gebundenheit jeglicher Bemühungen um Erweiterung von Kompetenzen und Handlungsspielräumen abhebt und im Kontrast der medialen Optionen deren Möglichkeiten und Grenzen auslotet, zielt letztere auf einen gedeihlichen Umgang mit konkreten Problemkonstellationen und Interaktionssituationen.

$\mathrm{Zu}$ guter Letzt bleiben sowohl die reflexiven, theoretisch motivierten als auch die praxisorientierten Bemühungen allzu oft auf lokale oder nationale Perspektiven beschränkt. Die Medienentwicklung zeichnet sich jedoch in vielerlei Hinsicht durch internationale und globale Dimensionen aus, denen auch in der Medienpädagogik Rechnung getragen werden muss (vgl. Volkmer 1995).

Die Modellierung und Beförderung von Medienkompetenz ist zur wichtigsten Zielorientierung der zeitgenössischen Medienpädagogik geworden. Dabei ist die Tragweite der Überlegungen nicht zu unterschätzen, solange Medienkompetenz nicht in verkürzter Weise verstanden oder gar auf algorithmisches Denken oder technische Fertigkeiten reduziert wird; denn im Anschluss an die Beobachtungen, die zur Rede von der Mediatisierung der Lebenswelt geführt haben, zeichnet sich ein paradigmatischer Wandel in der Medientheorie ab. Medialität ist keine optioneile Dimension, die zur Bestimmung von Erziehung, Bildung, Sozialisation, Kommunikation, Gesellschaft und Kultur quasi hinzukommen kann oder auch nicht, sie bezeichnet vielmehr die unausweichliche Verfasstheit dieser Bereiche. Im Zuge des „medial turn“ (vgl. Margreiter 1999; Weber 1999) stellen sich denn auch die Aufgaben der Medienpädagogik in neuer Perspektive. Die zentrale Herausforderung besteht dabei darin, die Suche nach pädagogischen Schonräumen jenseits medienimprägnierter Lebenswelten aufzugeben und aus dem Netz medialer Verstrickungen heraus Angebote zur kritischen Reflexion, Nutzung und Gestaltung von Bildungs- und Lernumgebungen zu machen. So gesehen erscheint die Frage nach der „Bildung in virtuellen Welten“ (vgl. Schindler/Bader/Eckmann 2001) in einem neuen Licht: „Brauchen wir ein neues Konzept von Bildung, ohne den alten Namen? Sagen wir etwa als Kompetenz zum Differenzmanagment? Als pragmatisch (noch) nicht festgelegtes Wissen? Als Überschussqualifikation? Als Medienlust und Entlarvung von Kontingenz durch Kontingenz? Als Kompetenz zum Vorbehalt gegen den Status quo? Als Variationskompetenz bei der Anwendung von Kulturprogrammen? Als Erinnerungskompetenz? Insgesamt als Differenzkompetenz zum beschleunigten Kompetenzverbrauch?“ (Schmidt 2002, S. 92). Schmidt (ebd.) schlägt probeweise vor, eine solche Kompetenz als Medienkompetenz zu bezeichnen. Ob diese als legitimes Nachfolgekonzept zu Bildung fungieren kann, wird noch zu diskutieren sein - plausibel ist der Vorschlag angesichts der Medienwirklichkeiten und der entsprechenden Orientierungs- und Gestaltungserfordernisse allemal. 


\section{Literatur}

Baacke, D.: Kommunikation und Kompetenz. Grundlegung einer Didaktik der Kommunikation und ihrer Medien. München 1973.

Baacke, D.: Die Welt als Clip?. In: Schorb, B./Stiehler, H.-J. (Hrsg.): Medienlust - Medienlast. Was bringt die Rezipientenforschung den Rezipienten? München 1996, S. 193-204.

Baacke, D.: Medienpädagogik. Tübingen 1997.

Baacke, D.: Die neue Medien-Generation im New Age of Visual Thinking. In: Gogolin, L/Lenzen D. (Hrsg.): Medien-Generation. Opladen 1999, S. 137-149.

Bayerische Landeszentrale für neue Medien: Medienkompetenz im Informationszeitalter. München 1996.

Dijk, T. A. van: Communicating Racism. Newbury Park 1987.

Enquete-Kommission „Zukunft der Medien in Wirtschaft und Gesellschaft“ - Deutschlands Weg in die Informationsgesellschaft (Hrsg.): Medienkompetenz im Informationszeitalter. Bonn 1997.

Hug, Th.: Lesarten des ,Instant Knowledge*. In: Ders. (Hrsg.): Technologiekritik und Medienpädagogik. Zur Theorie und Praxis kritisch-reflexiver Medienkommunikation. Baltmannsweiler 1998, S. 180-188.

Hug, Th.: Medienpädagogik - Begriffe, Konzeptionen, Perspektiven. In: Rusch, G. (Hrsg.): Einführung in die Medienwissenschaft. Wiesbaden 2002, S. 189-207.

Issing, L. J. (Hrsg.): Medienpädagogik im Informationszeitalter. Weinheim 1987.

Lauffer, J./Volkmer, I. (Hrsg.): Kommunikative Kompetenz in einer sich ändernden Medienwelt. Opladen 1995.

Margreiter, R.: Realität und Medialität. Zur Philosophie des ,Medial Tum*. In: Medien Journal. Zeitschrift für Kommunikationskultur, Jg. 23, H. 1,1999, S. 9-18.

Merkert, P. R.: Medien und Erziehung. Darmstadt 1992.

Merkert, P. R.: Medienpädagogik. In: Hierdeis, H./Hug, Th. (Hrsg.): Taschenbuch der Pädagogik, Bd. 3, Baltmannsweiler 1997 (5. Aufl.), S. 1057-1066.

Moser, H.: Einführung in die Medienpädagogik. Aufwachsen im Medienzeitalter. Opladen 1995.

Polanyi, M.: Implizites Wissen. Frankfurt/M. 1985.

Popper, K. R.: Objektive Erkenntnis. Ein evolutionärer Entwurf. Hamburg 1973 (1972).

Rein, A. von (Hrsg.): Medienkompetenz als Schlüsselbegriff. Bad Heilbmnn 1996.

Rickert, L.: Medien heute. Grundlagen - Forschung - Pädagogik. Ein annotiertes Literaturverzeichnis herausgegeben vom Institut Jugend Film Fernsehen. München 1993.

Rickheit, M.: Grundlagen einer kognitiven Wortsemantik. Opladen 1993.

Robertson, R.: Globalization - Social Theory and Global Culture. London 1992.

Rusch, G.: Erkenntnis, Wissenschaft, Geschichte. Von einem konstruktivistischen Standpunkt. Frankfurt/M. 1987.

Schindler, W./Bader, RTEckmann, B. (Hrsg.): Bildung in virtuellen Räumen. Praxis und Theorie außerschulischer Bildung mit Internet und Computer. Frankfurt/M. 2001.

Schmidt, S. J.: Kognitive Autonomie und soziale Orientierung. Konstruktivistische Bemerkungen zum Zusammenhang von Kognition, Kommunikation, Medien und Kultur. Frankfurt/M. 1994.

Schmidt, S. J.: Von Bildung zu Medienkompetenz - und retour? In: Kappus, H. (Hrsg.): Nützliche Nutzlosigkeit. Bildung als Risikokapital. Wien 2002, S. 63-96.

Schorb, B.: Stichwort .Medienpädagogik*. In: ZfE, Zeitschrift für Erziehungswissenschaft Jg. 1, H. 1,1998, S. 7-22. 
Schorb, B.: Vermittlung von Medienkompetenz als Aufgabe der Medienpädagogik. [WWW- Dokument] http://www.uni-leipzig.de/ schorb/mekomp.htm (Stand: 18.8.1999) 2000.

Schütz, A./Luckmann, Th.: Strukturen der Lebenswelt. Frankfurt/M. 1979/1984.

Schwarzer, R.: „Telelernen mit Multimedia in der Informationsgesellschaft“. In: Schwarzer, R. (Hrsg.): MultiMedia und TeleLearning. Lernen im Cyberspace. Frankfurt/New York 1998, S. 9-16.

Sinhart-Pallin, D.: Medienpädagogik. In: Bernhard, A./Rothermel, L. (Hrsg.): Handbuch Kritische Pädagogik. Weinheim 1997, S. 383-396.

Volkmer, I.: Von der Medienpädagogik zur Media Literacy - Kommunikative Kompetenz in einer internationalen Medienwelt. In: Lauffer, J./Volkmer, I. (Hrsg.): Kommunikative Kompetenz in einer sich ändernden Medienwelt. Opladen 1995, S. 179-185.

Volkmer, I.: ,Hic et nunc* von Nachrichtengenerationen. Überlegungen zu der Kategorie des .Daseins1 aus der Sicht globaler Phänomenologie“. In: Hug, Th. (Hrsg.): Technologiekritik und Medienpädagogik. Zur Theorie und Praxis kritisch-reflexiver Medienkommunikation. Baltmannsweiler 1998, S. 167-179.

Weber, St.: Die Welt als Medienpoiesis. Basistheorien für den .Medial Tum“. In: Medien Journal. Zeitschrift für Kommunikationskultur, Jg. 23, H. 1,1999, S. 3-8.

Wensiersky, H.-J. von: Medien- und Kulturpädagogik: Medienerziehung, Kulturarbeit, jugendkulturelle Bildung. In: Krüger, H.-H./Rauschenbach, Th. (Hrsg.): Einführung in die Arbeitsfelder der Erziehungswissenschaft. Opladen 1995, S. 159-176.

Winterhoff-Spurk, P.: Fernsehen und Weltwissen. Der Einfluss von Medien auf Zeit-, Raum- und Personenschemata. Opladen 1989. 